\title{
Plane strain tension fracture at high strain rate
}

\author{
Morwan Adlafi ${ }^{1,2}$, Bertrand Galpin ${ }^{1,2}$, Laurent Mahéo ${ }^{1,2}$, Christian C. Roth ${ }^{3}$, Dirk Mohr ${ }^{3}$, \\ and Vincent Grolleau ${ }^{*}, 1,3$
}

${ }^{1}$ Université Bretagne Sud, IRDL, UMR CNRS 6027, F-56100, Lorient, France

${ }^{2}$ Ecoles Saint-Cyr Coëtquid6n, CREC, F-56380 Guer, France

${ }^{3}$ Department of Mechanical and Process Engineering, ETH Zurich, Switzerland

\begin{abstract}
Under plane stress conditions, most micromechanical and phenomenological models predict a minimum in ductility for plane strain tension stress state. Therefore, the stress state of plane strain tension plays a crucial role in many forming and crash applications and the reliable measurement of the strain to fracture for plane strain tension is particularly crucial when calibrating modern fracture initiation models. Recently, a new experimental technique has been proposed for measuring the strain to fracture for sheet metal after proportional loading under plane strain conditions. The basic configuration of the new setup includes a dihedral punch which applies out-of-plane loading onto a Nakazima-type of discshaped specimen with two symmetric holes and an outer diameter of $60 \mathrm{~mm}$. In the present work, the applicability of the test is extended to high strain rates. High strain rates of about 100/s to 200/s are obtained using a drop weight tower device with an original sensor for load measurements. Quasi static tests are also performed for comparison, keeping the same specimen geometry, image recording parameters and set-up. The effective strains at fracture are compared from quasi-static to high strain rate loading for three different materials, i.e one aluminium alloy and two steels.
\end{abstract}

\section{Introduction}

The plane-strain-tension stress state plays a crucial role in many drawing applications as well as in crash events. Under plane stress conditions, most micro-mechanical and phenomenological models predict a minimum in ductility for a plane strain tension stress state. Forming Limit Curves (FLC) or modern stress state dependent fracture initiation models exhibit a "plane strain ductility valley" between uniaxial and equi-biaxial tension. Hence, the reliable measurement of the strain to fracture for plane strain tension is of utmost importance, which requires reliable and accurate experimental techniques. Attempts to classify the wide range of proposed experimental techniques can be found in Cheong et al. [1] or Grolleau et al. [2]. As already observed by Ghosh and Hecker [3], Morales et al. [4], and more recently by Tharrett and Stoughton [5], bending strain has a positive influence,

*Corresponding author: vgrolleau@ethz.ch 
effectively delaying through thickness necking. The V-bending test, as proposed in the VDA 238-100 [6] standard and used by Roth et al. [7], leads to a bending dominated state. In this case, cracks occur on the free surface and the strain path to fracture is almost free from any change related to tensile instability. The major drawback of V-bending is its maximum obtainable strain, limited by geometrical constraints, i.e. "fold over" [7]. This hinders its use for thin and/or highly ductile materials (e.g. Grolleau et al. [2] and Noder et al. [8]) without an initial pre-straining of the specimen. Usually pre-straining of the material is performed under stress states different from PST, leading to non-proportional loading histories, which may influence the obtained fracture strain. In contrast, the dual-hole mini-Nakazima test with a dihedral punch (Fig. 1a), creates a constant stress state [2]. It is composed of a disc-shaped specimen (component (1) Fig. 1) with an outer diameter of $60 \mathrm{~mm}$ and two $8 \mathrm{~mm}$ diameter circular cut-outs (2) that form a $10 \mathrm{~mm}$ long central ligament. The cut-outs on the specimen top face, opposite to the punch, are chamfered to prevent early fracture from the edge of the holes. The dihedral punch (component (3) ) has a $90^{\circ}$ tip angle and a $1 \mathrm{~mm}$ edge radius. With a so-called inverse configuration, where the specimen (component (1)) is pushed against the stationary dihedral punch (component (3)), the displacement field of the specimen surface can be monitored using Digital Image Correlation (DIC). This test leads to high major-tominor strain ratios (above 20) and a homogenous plane strain tension stress state along $80 \%$ of the ligament length.

In this study, we present the use of the dual hole plane strain tension mini Nakazima test with dihedral punch (DMN) adapted to a drop-tower apparatus to perform experiments at high strain rates in the range of several 100/s. We compare fracture strain measurements from high and low strain rate experiments on a $1.5 \mathrm{~mm}$ thick AA2024 aluminium alloy and two DP steels, a $0.8 \mathrm{~mm}$ thick DP450 and a $1.5 \mathrm{~mm}$ thick DP600.

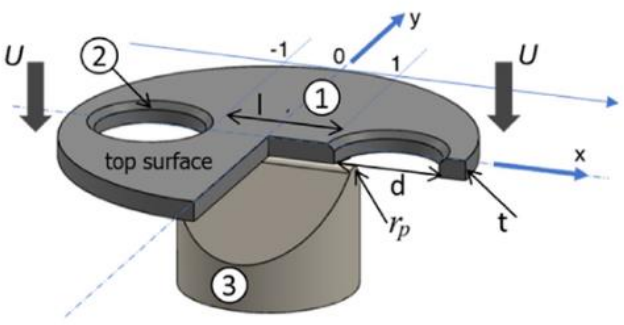

(a)

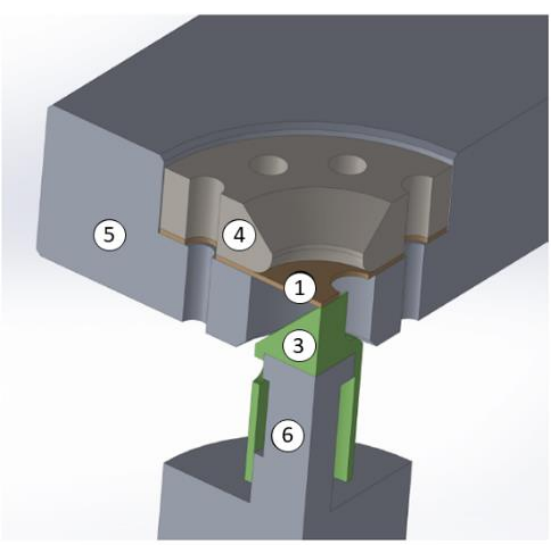

(b)

Fig. 1. Schematic view of the dual hole mini Nakazima type specimen (1) and the dihedral punch (3) (a). Quarter view of the specimen inserted on the moving crosshead (5) of a drop weight tower with the dihedral punch (3) covering the sensing part of the load cell (6).

\section{Experimental set-up}

The Fig. $1 \mathrm{~b}$ shows a quarter view of the device. The specimen is clamped between the moving crosshead (component (5)) and the upper die (component (4)). The dihedral punch is a cap, covering the sensing part of a specially designed gauge load cell (component (6), see [10]). The experimental set-up is partially shown in Fig. 2 in its initial state just before testing. Two 
1Mpx high-speed cameras Photron SA5 (component (8)) equipped with $105 \mathrm{~mm}$ focal length lenses (component (7)) capture the free surface of the specimen at a frame rate of $50 \mathrm{kHz}$ and an image size of $512 \times 272 \mathrm{px}$, leading to a spatial resolution of about $28 \mu \mathrm{m} / \mathrm{px}$. During the test, the focal distance between the cameras and the specimen is kept constant at about $300 \mathrm{~mm}$. The free flying stroke of the $20 \mathrm{~kg}$ crosshead is about $150 \mathrm{~mm}$, the speed of the specimen at onset of contact with the punch is about $1 \mathrm{~m} / \mathrm{s}$, and up to $3 \mathrm{~m} / \mathrm{s}$ if pre-tensioned springs are used to accelerate the crosshead. The initial speed is measured with two optical sensors, while the load is measured using a specially designed gauge load cell as shown in Galpin et al. [10] which is validated on Split Hopkinson Pressure Bar (SHPB) tests. An accelerometer is attached to the crosshead and a displacement sensor, Bullier M25L10, is used to measure the crosshead displacement with an accuracy of about $0.02 \mathrm{~mm}$ at a frequency of $125 \mathrm{kHz}$ using a triangulation principle.
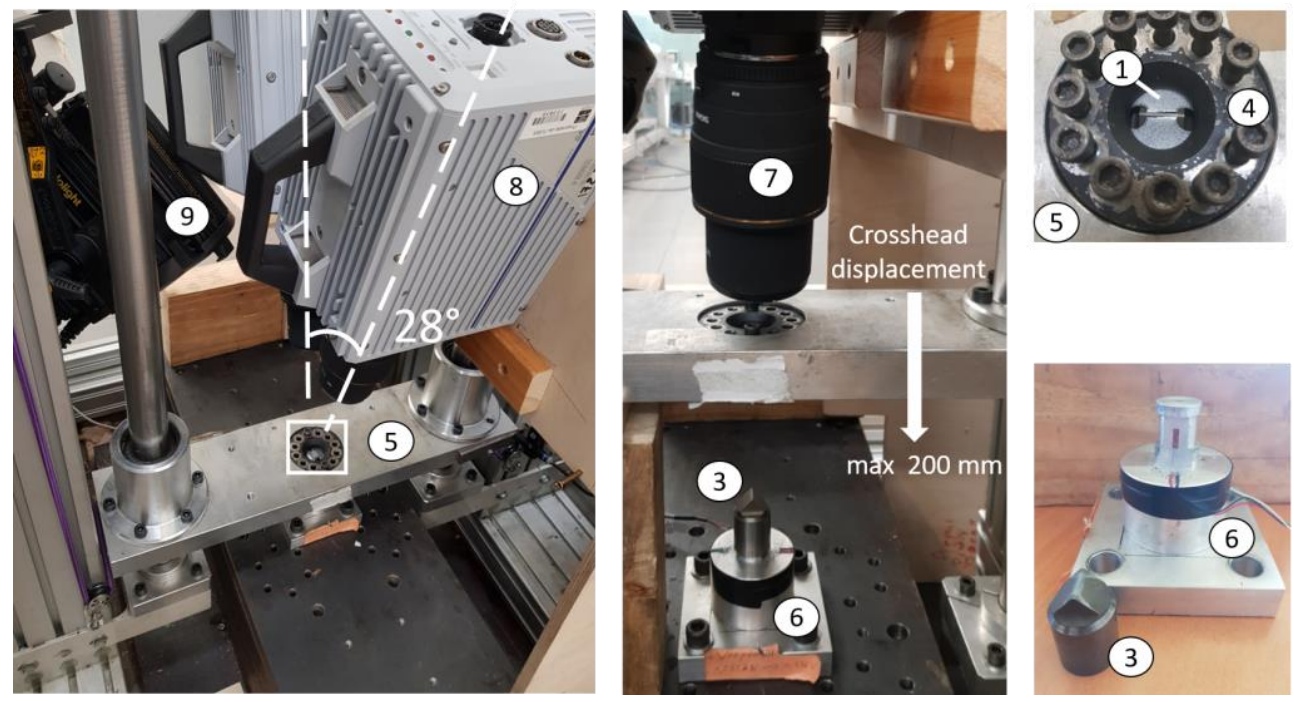

Fig. 2. The experimental drop weight tower set up showing the two high-speed cameras (component (8)), lenses (7), and the $28^{\circ}$ angle between the focal axis. The specimen (1) is clamped between the moving crosshead (5) and the upper die component (4). The sensing extension of the load cell (6) is covered with the dihedral cap (3).

When performing low strain rate DMN tests, due to the higher resolution of the low speed cameras, the spatial resolution of the specimen surface is about $10 \mu \mathrm{m} / \mathrm{px}$ (see for example [2]). In conjunction with a subset size of $28 \times 28 \mathrm{px}$ and a step size of $6 \mathrm{px}$ this has been proven to allow the accurate measurement of effective strains up to 1.0 from DIC analysis in the ligament area of the DMN (with a noise floor on the effective strain calculation of about $7.5 \mathrm{e}-$ 4 [-] (standard deviation about 2.5e-4)).

In order to validate the high speed camera set-up for DIC, previous quasi-static tests from the literature [2] are reproduced with the present lower resolution set-up on the AA2024 aluminium alloy, the DP450 and DP600 steels. Figure 3 shows a comparison of two recorded pictures of the ligament area from the low and high speed camera setup. When using high speed cameras, 3 different subset sizes are tested, i.e. 9, 12 and 14 px, with a constant step size of $4 \mathrm{px}$. While increasing the subset size has a positive influence on avoiding loss of correlation, decreasing the subset size from 14 to $9 \mathrm{px}$ is found to be necessary in order to increase the maximum effective strains which can be calculated in the DMN setup from 0.3 
to 1.0. Choosing the subset size according to the camera system resolution used, the use of a high speed camera or low speed camera system will yield identical measurements.
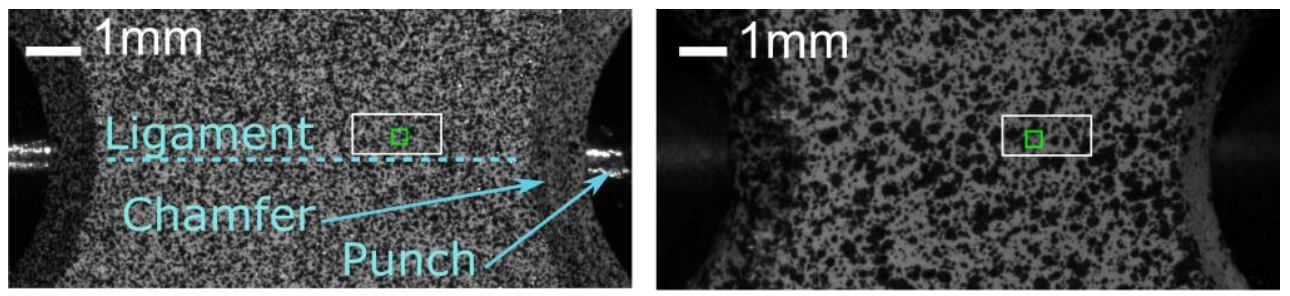

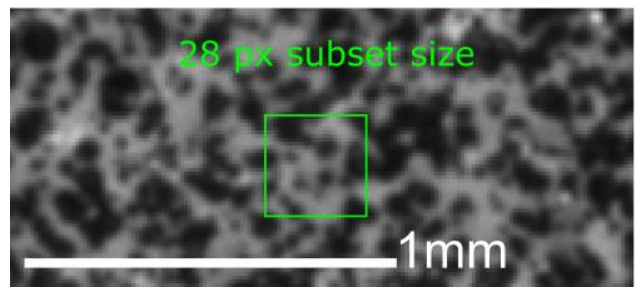

Quasi-static test, $10 \mu \mathrm{m}$ pixel size

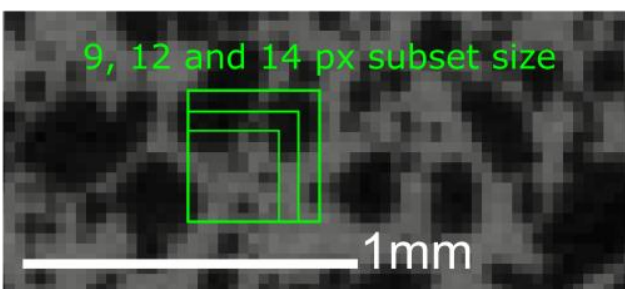

High strain rate set-up, $28 \mu \mathrm{m}$ pixel size

Fig. 3. Comparison of recorded images from quasi-static (left) and high strain rate set-ups (right). Original images are plotted on top, the ligament is indicated with a dashed light blue line. The respective close up views (white boxes) are presented on the bottom, along with the subset size (green lines).

\section{Experimental results}

An image of the top surface of a DP600 specimen at the onset of fracture during the drop weight tower test is presented in Fig. 4a. A through thickness optical micrograph of a specimen obtained from a slow test interrupted close to the force maximum is presented in Fig. $4 \mathrm{~b}$. The ligament direction is perpendicular to the figure, with the location of the $1 \mathrm{~mm}$ punch radius illustrated by a green circle. Crack initiation occurred on the free surface and the crack has already propagated through more than half the thickness.

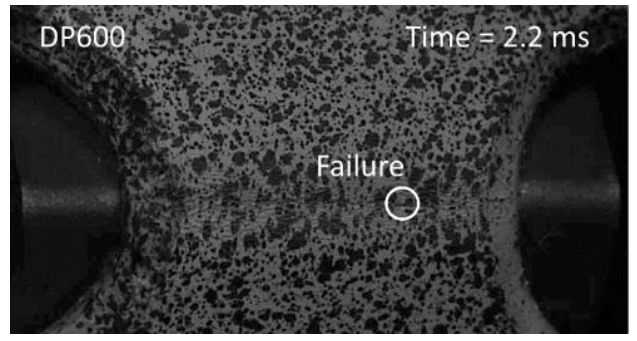

(a)

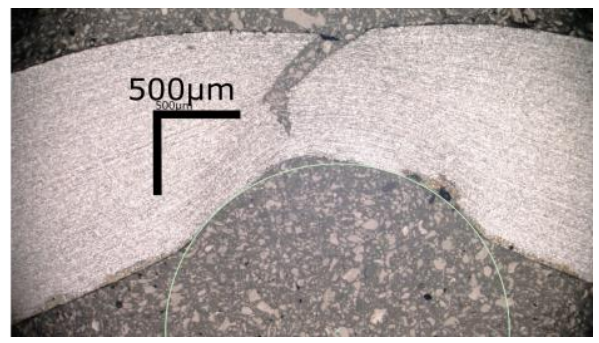

(b)

Fig. 4. Free surface of a DP600 specimen at fracture (a). Through thickness optical micrograph of a DP600 specimen from an interrupted quasi-static test just before force maximum.

Figure 5 shows the force versus time evolution for the three materials obtained from the drop weight tower tests at a speed of $1 \mathrm{~m} / \mathrm{s}$, corresponding to an initial strain rate of about 200/s. The onset of fracture always occurs close to the force maximum. The strain rate evolution is presented as a function of time for the DP600 material (Fig. 5b). While the strain rate evolution starts with a plateau of 0.2 in effective strain for all materials, it subsequently 
increases by a factor up to 4 (2) at an effective strain level of about $0.7(0.3)$ for the dual phase steels (and AA2024 respectively).

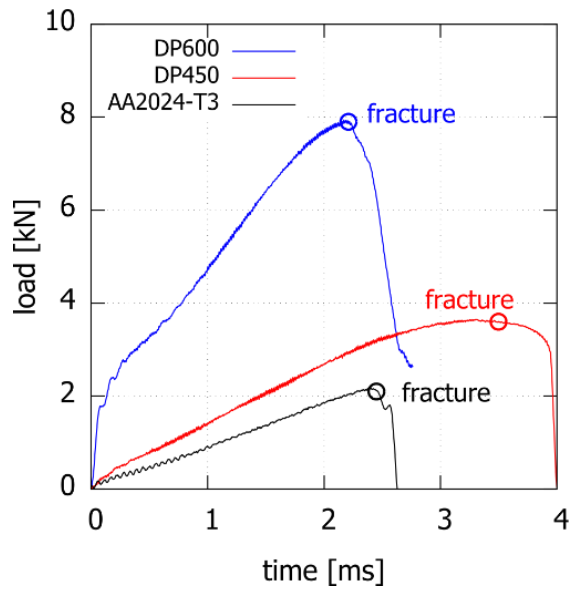

(a)

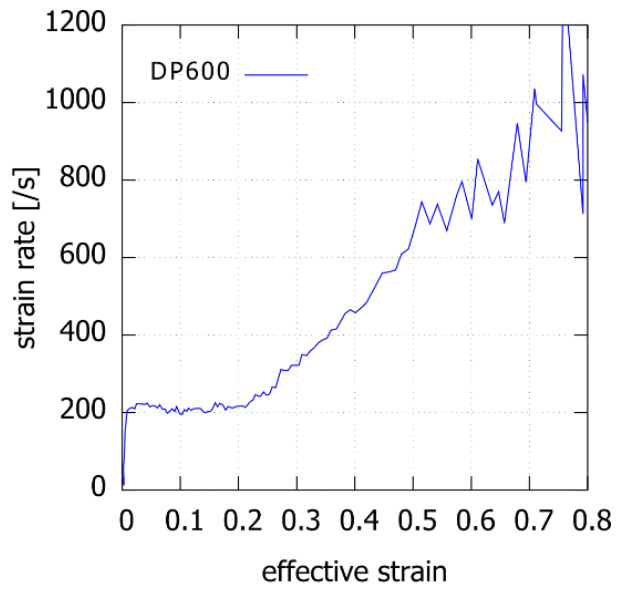

(b)

Fig. 5. Load evolution as a function of time during drop weight tower tests (a), and strain rate evolution in the case of DP600 steel (b).

Figure 6 shows a comparison of the calculated effective strain fields in the case of the DP600 steel during high strain rate and quasi-static tests. Both pictures are taken at the onset of fracture on the free surface of the specimen, at load values of about $7 \mathrm{kN}$ and $8 \mathrm{kN}$ for low and high strain rate tests, respectively. For the DP600 the calculated effective strains at the location of the first crack are $0.81 \pm .02$ for all tests, irrespective of the speed and for all 3 repeats. A similar result is obtained for the DP450 steel, with a fracture strain about $0.84 \pm 0.03$. For the AA2024-T4 aluminium alloy, the effective fracture strain at low strain rates is $0.27 \pm 0.02$, while a fracture strain about $0.32 \pm 0.02$ is calculated for high strain rates.

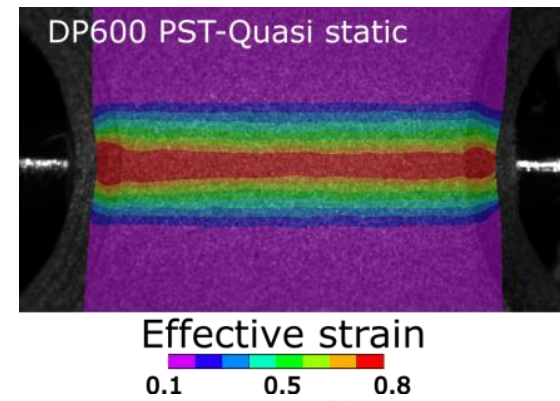

(a)
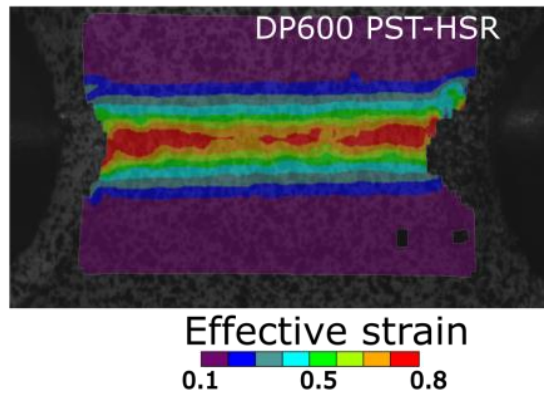

(b)

Fig. 6. Comparison of calculated effective strain fields from quasi-static experiment (a), and high strain rate tests (b).

It is noteworthy that the main advantages of the DMN at low strain rates are kept for experiments at strain rates from 200 to $1000 / \mathrm{s}$. Along the ligament, a very homogenous strain field prevails. For example, for the DP 600, at onset of fracture, the effective strain along the ligament varies only in the range [0.75:0.85]. At the same time, the DMN maintains a plane strain tension stress state throughout the test, with a longitudinal to transverse surface strain ratio above 20 for the central $80 \%$ of the ligament length. 


\section{Conclusion}

The use of the dual hole plane strain tension mini Nakazima test with dihedral punch (DMN) is extended for high strain rates by means of a drop weight tower test setup. Plane strain tension tests are performed on an aluminium alloy AA2024-T3 and two dual phase steels, i.e. a DP450 and a DP600. Digital Image Correlation on images obtained at high speeds shows that the main advantages of the DMN are preserved from slow up to high strain rates of several hundred per second. For the two dual phase steels, a constant effective strain at fracture under plane strain tension is observed. In contrast, an increase in the fracture strain from 0.27 at low strain rates $(\sim 0.001 / \mathrm{s})$ to 0.32 at elevated strain rates (from about $200 / \mathrm{s}$ to $350 / \mathrm{s}$ during the test) is observed for the AA2024-T3 aluminium alloy.

\section{Acknowledgments}

This work was financially supported by French company Safran; and Fondation Saint Cyr Coëtquidan. The authors would like to take this opportunity of expressing their sincere appreciation for these funds. Mr. Erik de Best (ETH Zurich) and Mr. Knut Krieger (inspire $\mathrm{AG})$ are gratefully acknowledged for their help with the experiments.

\section{References}

1. Cheong, K., Omer, K., Butcher, C., George, R., \& Dykeman, J. (2017). Evaluation of the VDA 238-100 Tight Radius Bending Test using Digital Image Correlation Strain Measurement. Journal of Physics: Conference Series, 896.

2. Grolleau, V., Roth, C. C., Lafilé, V., Galpin, B., \& Mohr, D. (2019). Loading of miniNakazima specimens with a dihedral punch: Determining the strain to fracture for plane strain tension through stretch-bending. International Journal of Mechanical Sciences, 152.

3. Ghosh, A. K., \& Hecker, S. S. (1974). Stretching limits in sheet metals: in-plane versus out-of-plane deformation. Metallurgical Transactions, 5.

4. Morales-Palma, D., Vallellano, C. and García-Lomas, F.J., 2013. Assessment of the effect of the through-thickness strain/stress gradient on the formability of stretch-bend metal sheets. Materials \& Design, 50.

5. Tharrett, M. R., \& Stoughton, T. B. (2003). Stretch-bend forming limits of 1008 AK steel (No. 2003-01-1157). SAE Technical Paper.

6. VDA 238-100, (2017). Test Specification: Plate Bending Test for Metallic Materials

7. Roth, C. C., \& Mohr, D. 2016. Ductile fracture experiments with locally proportional loading histories. International Journal of Plasticity, 79.

8. Noder, J., Abedini, A., \& Butcher, C. (2020). Evaluation of the VDA 238-100 tight radius bend test for plane strain fracture characterization of automotive sheet metals. Experimental Mechanics, 60.

9. B. Galpin , V. Grolleau, S. Umiastowski , G. Rio \& L. Mahéo (2008). Design and application of an instrumented projectile for load measurements during impact. International Journal of Crashworthiness, 13. 